\title{
Respuesta de indicadores nutricionales en vaquillas suplementadas con semillas de soja y algodón en Chaco, Argentina
}

\author{
Koza, G.A.; Mussart, N.B.; Fioranelli, S.A.; Álvarez Chamale, G.M.; Coppo, J.A. \\ Cátedra de Fisiología, Facultad de Ciencias Veterinarias, UNNE, Sargento Cabral 2139, Corrientes (3400), \\ Argentina. Tel. 03783-425753. E-mail: fisiologia@vet.unne.edu.ar.
}

\begin{abstract}
Resumen
Koza, G.A.; Mussart, N.B.; Fioranelli, S.A.; Álvarez Chamale, G.M.; Coppo, J.A.: Respuesta de indicadores nutricionales en vaquillas suplementadas con semillas de soja y algodón en Chaco, Argentina. Rev. vet. 20: 1, 15-21, 2009. El objetivo del ensayo fue comparar los efectos de las semillas enteras de algodón y soja como suplementos energéticoproteicos invernales en vaquillas cruza cebú sobre pasturas naturales del nordeste argentino. Noventa vaquillas de alrededor de $180 \mathrm{~kg}$ de peso se dividieron en 3 lotes de 30 ejemplares cada uno (10 Braford, 10 Brangus y 10 Limusin), a saber: lote SS (suplementado con semillas de soja: $2,60 \mathrm{~kg} / \mathrm{animal} / \mathrm{día}$ ), SA (con semillas de algodón: $2,40 \mathrm{~kg} / \mathrm{animal} / \mathrm{día}$ ) y CN: control sin suplemento. Durante el primer y segundo inviernos (126 y 131 días), mensualmente fue evaluado el peso, signos clínicos e indicadores hemáticos de estado nutricional. En promedio, los pesos finales fueron de $238,72 \mathrm{~kg}$ en SS, $236,10 \mathrm{~kg}$ en SA y $218,52 \mathrm{~kg}$ en $\mathrm{CN}(\mathrm{p}=0,04)$. Entre las distintas razas no se registraron diferencias significativas de peso ni parámetros de laboratorio. Las vaquillas suplementadas revelaron favorables aumentos de variables de los perfiles eritrocitario, proteico, lipídico y mineral. SA indujo los más altos valores de hematocrito y magnesio (deseables), así como de colesterol total, AST y GGT (indeseables), en tanto que SS generó los niveles más elevados de urea (deseable) y LDH (indeseable). Las elevaciones de lípidos y enzimas son relacionadas a una ligera sobrecarga hepática, la cual no repercutió en el estado general de salud de ningún animal. Tal ausencia de efectos secundarios de nivel clínico, aunada al aumento de peso y la mejoría de varios componentes del medio interno, justifican la utilización de las semillas oleaginosas ensayadas como suplemento energético-proteico invernal de ganado en crecimiento en la zona chaqueña de Argentina. El estudio comparativo efectuado no permite preferenciar un suplemento sobre el otro, pudiendo dirimirse la opción a través del beneficio económico devengado por cada uno de los tratamientos.
\end{abstract}

Palabras clave: vaquilla, suplementación invernal, semillas de algodón y soja, ganancia de peso, parámetros bioquímicos.

\begin{abstract}
Koza, G.A.; Mussart, N.B.; Fioranelli, S.A.; Álvarez Chamale, G.M.; Coppo, J.A.: Changes in nutritional indicators in heifers supplemented with soy and cotton seeds in Chaco, Argentina. Rev. vet. 20: 1, 15-21, 2009. The objective of this trial was to compare the effects of soy and cotton whole seeds, used as energy-protein winter supplements in half bred Zebu heifers, fed on natural pastures of Northeast Argentina. Ninety heifers around $180 \mathrm{~kg}$ liveweight were divided in 3 lots of 30 animals each (10 Braford, 10 Brangus, and 10 Limusin): lot SS (supplemented with soy seeds: $2.60 \mathrm{~kg} / \mathrm{animal} / \mathrm{day}$ ), lote SA (supplemented with cottonseed: $2.40 \mathrm{~kg} /$ animal/day) and lot $\mathrm{CN}$ : control without supplement. During first and second winters (126 and 131 days, respectively), liveweight, clinical signs, and hematic indicators of nutritional state were monthly evaluated. On average, final liveweights were $238.72 \mathrm{~kg}$ in $\mathrm{SS}, 236.10 \mathrm{~kg}$ in SA, and $218.52 \mathrm{~kg}$ in $\mathrm{CN}(\mathrm{p}=0.04)$. No significant differences in liveweight and laboratory parameters were registered among breeds). Favorable increases of variables of erythrocytary, proteic, lipidic and mineral profiles were verified in supplemented heifers. The highest values of hematocrit and magnesium (desirable), as well as the highest concentration of total cholesterol, AST and GGT (undesirable) were induced by SA, whereas SS generated the highest levels of urea (desirable) and LDH (undesirable). Lipidic and enzymatic increases are related to a slight hepatic overload, which did not have effect in health general state of any animal. This absence of secondary clinical effects, together
\end{abstract}


with liveweight increment and the improvement of several internal environment components, justify the use of the oleaginous seeds as energy-protein winter supplement in growing livestock from Chaco, Argentina. This comparative study does not indicate whether supplement is better than the other. Perhaps the latter should be decided taking into account economic matters related to each treatment.

Key words: heifer, winter supplementation, cotton and soy seeds, liveweight gain, biochemical parameters.

\section{INTRODUCCIÓN}

Durante el invierno las pasturas naturales disminuyen en cantidad y calidad, pudiendo ocasionar deficiencias energético-proteicas al ganado. Por su estacionalidad, las forrajeras tropicales no poseen suficiente calidad nutricional como para lograr la máxima producción de los rumiantes en pastoreo. El desequilibrio de nutrientes conlleva grandes inconvenientes en la producción animal ${ }^{1,15}$.

La deficiencia sistemática de la producción de forraje invernal imperante en nuestra zona, afecta el desarrollo y repercute en el peso de los bovinos en crecimiento, principalmente en las vaquillas de reposición ${ }^{8}$. La suplementación es una técnica de nutrición en activo desarrollo en toda la región subtropical, que permite adicionar los nutrientes que son deficitarios en las praderas, para mejorar la eficiencia de utilización del forraje, siendo una de las principales herramientas para la intensificación y diversificación de los modelos productivos tradicionales ${ }^{26}$.

La semilla de algodón (SA) es un alimento de gran valor nutritivo para el ganado, al aportar elevada cantidad de energía y proteínas (extracto etéreo $22,7 \%$, proteína bruta $19,1 \%$ ). Lamentablemente SA posee principios tóxicos como gosipol y ácidos grasos ciclopropenoides, capaces de alterar la salud del bovino, especialmente en la joven edad 9, 17 . En igual sentido, la semilla de soja (SS) y sus harinas derivadas pueden constituir un buen suplemento para cubrir los requerimientos nutricionales de los bovinos de carne en la región de pasturas tropicales ${ }^{6}$. Desafortunadamente también posee principios tóxicos, tales como anti-tripsina y factores anti-tiroideos, además de un elevado contenido de grasa (20\%) altamente insaturada (54 y $84 \%$ de ácidos linoleico y linolénico respectivamente) ${ }^{29}$. El exceso de lípidos dietarios ingeridos durante largos períodos puede generar daño hepático y afectar la salud del bovino ${ }^{12}$.

El objetivo del presente trabajo fue comparar los efectos de la suplementación energético-proteica invernal en base a semillas enteras de algodón y soja, sobre el peso vivo y algunos parámetros hematológicos y bioquímicos de vaquillas de reposición cruza cebú, bajo las condiciones imperantes en el nordeste argentino.

\section{MATERIAL Y MÉTODOS}

Las tareas de campo se realizaron en un establecimiento próximo a la localidad de San Bernardo, Chaco. Noventa vaquillas destete de aproximadamente $180 \mathrm{~kg}$ de peso vivo (PV) fueron distribuidas aleatoriamente en tres lotes de 30 ejemplares cada uno (10 de raza Braford +10 de raza Brangus +10 Cruza Limusin). Cada lote fue sometido durante su primer y segundo inviernos (126 y 131 días respectivamente), a una suplementación energético-proteica en base a semillas oleaginosas, sobre potreros de pastizal natural.

La distribución fue: lote $\mathrm{CN}$ (control sin suplemento), lote SS: suplementado con semillas de soja $(2,60$ $\mathrm{kg} / \mathrm{animal} / \mathrm{d}$ á; $1,4 \% \mathrm{PV}$ promedio) y lote SA: suplementado con semillas de algodón $(2,40 \mathrm{~kg} / \mathrm{animal} / \mathrm{d}$ á; $1,3 \%$ PV promedio). En la Tabla 1 se consigna el resultado del análisis de los componentes nutricionales de los suplementos empleados a lo largo de la experiencia, determinaciones realizadas el en Laboratorio de Química Agrícola de la Facultad de Ciencias Agrarias, UNNE. La disponibilidad forrajera promedio a lo largo del ensayo fue de 12.000 a $15.000 \mathrm{~kg} \mathrm{MS} /$ hectárea. Mensualmente se practicó la rotación de potreros. El suplemento fue suministrado a los animales en horario vespertino (5 PM).

Durante el lapso de suplementación invernal, con periodicidad mensual se efectuaron pesajes individuales (sin desbaste, en báscula), exámenes clínicos (de visu) y extracciones de sangre por venopunción yugular a 15 animales de cada lote. Las muestras de sangre se fraccionaron en dos alícuotas, una fue tratada con anticogulante (EDTA) para la determinación de variables hematológicas (hematocrito, hemoglobina, concentración de eritrocitos e índices hematimétricos VCM, HCM y CHCM), empleándose un analizador hematológico electrónico. La sangre restante fue centrifugada para separar el suero, a partir del cual se obtuvieron las concentraciones de proteínas totales (PT), albúmina (AL), globulinas (GL), relación albúmina/globulinas (AG), urea (UR), colesterol total (CT), triglicéridos (TG), lipoproteínas beta y alfa (LB, LA y relación entre ambas: BA), colesterol ligado a dichas lipoproteínas (C-LDL, C-HDL), aspartato aminotransferasa (AST), gamma glutamil transferasa (GGT), lactato dehidrogenasa $(\mathrm{LDH})$ y valores del ionograma $(\mathrm{Ca}, \mathrm{P}, \mathrm{Mg}, \mathrm{Na}$, 
$\mathrm{K}, \mathrm{Fe}, \mathrm{Cu}$ ), mediante espectrofotometría, fotometría de llama y electroforesis (reactivos Wiener Lab).

Los resultados fueron analizados como medidas repetidas por medio del software Statistica 2002. A través del análisis de la variancia (ANOVA) se investigaron los efectos tratamiento (tipo de suplemento) y tiempo (ontogenia), así como la interacción entre ambos. La comparación de medias se realizó por el test de Duncan.

\section{RESULTADOS Y DISCUSIÓN}

El peso vivo de los animales bajo ensayo fue influido significativamente por el tratamiento $(\mathrm{p}=$ $0,048)$; al culminar los ensayos el lote SA registró $236,10 \mathrm{~kg}$, el lote SS $238,72 \mathrm{~kg}$ y el lote CN 218,52 $\mathrm{kg}$. La raza no ejerció modificaciones significativas sobre el peso ni los parámetros de laboratorio considerados. En ningún animal se detectaron síntomas de efectos secundarios atribuibles a las semillas ensayadas.

Otros autores, comparando vaquillas divididas en un lote control y otro experimental suplementado con semilla de algodón, registraron en este último significativos incrementos de peso tanto en el primero como en el segundo año de ensayos, así como una considerable mejoría de la condición corporal ${ }^{28}$. En cambio, al comparar los efectos de la semilla de soja versus harina de soja suministradas a vacas de diferentes edades, no pudieron demostrarse diferencias significativas en las ganancias de peso ni cambios de condición corporal ${ }^{7}$.

El hematocrito resultó significativamente más elevado en SA (39\%) que en SS (36\%) y CN (36\%), p = 0,043 . El efecto tiempo también afectó de manera significativa a esta variable $(\mathrm{p}=0,0001)$. La hemoglobina solamente reveló una tendencia al aumento en sendos lotes suplementados $(\mathrm{p}=0,07)$, registrando $14,50 \mathrm{~g} / \mathrm{dl}$ en SA, 12,71 g/dl en SS y 12,60 g/dl en CN. Los índices hemoglobínicos HCM y CHCM se modificaron significativamente por el efecto tiempo $(p=0,018$ y $p=0,0001$ respectivamente), en tanto que la tasa de eritrocitos y su volumen corpuscular medio (VCM) no sufrieron variaciones significativas relacionadas a los efectos tratamiento ni tiempo.

En novillos cruza cebú suplementados con semilla de algodón se lograron niveles de hematocrito aún más elevados, de 40 a $45 \%{ }^{10}$. Seguramente tales aumentos respondan al aporte de oligoelementos eritropoyéticos efectuado por el suplemento; en tal sentido, la administración de hierro y cobre produjo significativas elevaciones de hematocrito, hemoglobina, eritrocitos y VCM en terneros ${ }^{20}$. No obstante, excesiva cantidad de semilla de algodón en la dieta puede afectar negativamente los valores del hemograma debido a la acción nociva del pigmento polifenólico gosipol ${ }^{17}$. El ambiente subtropical quizás influya en algunos de los valores del hemograma bovino, pues en condiciones de elevadas temperatura y humedad ambiental se registraron au-
Tabla 1. Composición nutricional de los suplementos empleados.

\begin{tabular}{lccc}
\hline componente & algodón (1) & algodón (2) & soja \\
\hline materia seca (\%) & 90,23 & 92,25 & 88,61 \\
proteína bruta (\%) & 17,51 & 19,26 & 29,90 \\
extracto etéreo (\%) & 13,56 & 13,98 & 13,29 \\
fibra bruta (\%) & 39,12 & 40,37 & 13,79 \\
fibra detergente neutro (\%) & 66,40 & 68,5 & 30,10 \\
fibra detergente ácido (\%) & 53,84 & 56,89 & 21,95 \\
energía digestible (Mcal/kg) & 2,18 & 2,56 & 3,31 \\
\hline
\end{tabular}

(1) y (2): primer y segundo año de ensayos.

Tabla 2. Efectos del tratamiento y del tiempo sobre variables relacionadas al perfil nitrogenado de las vaquillas.

\begin{tabular}{ccccccc}
\hline tratamiento & tiempo & $\mathrm{PT}(\mathrm{g} / \mathrm{dl})$ & $\mathrm{AL}(\mathrm{g} / \mathrm{dl})$ & $\mathrm{GL}(\mathrm{g} / \mathrm{dl})$ & $\mathrm{AG}$ & $\mathrm{UR}(\mathrm{g} / \mathrm{l})$ \\
\hline \multirow{2}{*}{$\mathrm{SA}$} & 1 & 6,78 & 3,41 & 3,38 & 1,03 & 0,45 \\
& 2 & 6,89 & 3,62 & 3,26 & 1,16 & 0,39 \\
$\mathrm{SS}$ & 1 & 6,73 & 3,47 & 3,25 & 1,10 & 0,54 \\
& 2 & 6,92 & 3,59 & 3,33 & 1,12 & 0,37 \\
$\mathrm{CN}$ & 1 & 6,77 & 3,29 & 3,48 & 0,97 & 0,44 \\
& 2 & 6,81 & 3,48 & 3,34 & 1,06 & 0,39 \\
$\mathrm{EE}$ & & 0,129 & 0,079 & 0,128 & 0,055 & 0,017 \\
$\mathrm{p}$ & & 0,0002 & 0,0003 & 0,183 & 0,102 & 0,0001 \\
\hline
\end{tabular}

SA: semilla de algodón, SS: semilla de soja, $\mathrm{CN}$ : control sin suplemento, EE: error estándar, p: significancia, 1 y 2: primer y segundo inviernos, PT: proteínas totales, AL: albúmina, GL: globulinas, AG: relación AL/GL.

mentos de la concentración de hemoglobina, aunque no fue afectada la cantidad de eritrocitos circulantes ${ }^{31}$.

En la Tabla 2 se consignan las variaciones del perfil nitrogenado atribuibles a los efectos tratamiento y tiempo, a lo largo de los dos inviernos en que los animales recibieron suplementación. El efecto tiempo accionó significativamente sobre todas las variables del perfil nitrogenado. En cuanto al efecto tratamiento, surge que éste no modificó las concentraciones séricas de PT ni GL. En cambio, los valores de AL de los lotes SA (3,52 $\mathrm{g} / \mathrm{dl})$ y SS $(3,53 \mathrm{~g} / \mathrm{dl})$ fueron significativamente más altos $(\mathrm{p}=0,019)$ que los del lote $\mathrm{CN}(3,38 \mathrm{~g} / \mathrm{dl})$. Consecuentemente, la relación $\mathrm{AG}$ fue significativamente influenciada por el tratamiento $(1,10$ para $\mathrm{SA} ; 1,11$ para SS y 10,1 para $\mathrm{CN}$ ).

Los valores de PT obtenidos en esta experiencia para los lotes SA, SS y CN $(6,84 ; 6,82$ y 6,79 g/dl respectivamente) se aproximan a los citados como normales por algunos autores $(6,6 \mathrm{a} 9 \mathrm{~g} / \mathrm{dl}){ }^{16}$ aunque se ubican algo por debajo a los referidos por otros $(7,4$ a 7,9 g/dl) 12. Los niveles de AL y GL se encuentran dentro del intervalo de referencia admitido para los bovinos ${ }^{12,16}$. En ensayos anteriores no se verificaron modificaciones significativas de PT ni AL en vacunos alimentados con raciones isoenergéticas-isoproteicas a base de semilla de algodón y sorgo ${ }^{21}$. Los lípidos de la semilla de algodón no influirían sobre los niveles proteicos del suero del bovino pues la concentración de PT de vacas en distintas etapas del período de lactancia, no difirió en- 
Tabla 3. Efectos del tratamiento sobre variables relacionadas al perfil lipídico de las vaquillas.

\begin{tabular}{ccccc}
\hline tratamiento & CT $(\mathrm{g} / \mathrm{l})$ & $\mathrm{TG}(\mathrm{g} / \mathrm{l})$ & $\mathrm{C}-\mathrm{HDL}(\mathrm{g} / \mathrm{l})$ & $\mathrm{C}-\mathrm{LDL}(\mathrm{g} / \mathrm{l})$ \\
\hline $\mathrm{SA}$ & $2,37 \mathrm{a}$ & $0,38 \mathrm{a}$ & $1,48 \mathrm{a}$ & $0,46 \mathrm{a}$ \\
$\mathrm{SS}$ & $2,24 \mathrm{a}$ & $0,38 \mathrm{a}$ & $1,40 \mathrm{a}$ & $0,45 \mathrm{a}$ \\
$\mathrm{CN}$ & $1,34 \mathrm{~b}$ & $0,21 \mathrm{~b}$ & $0,92 \mathrm{~b}$ & $0,24 \mathrm{~b}$ \\
$\mathrm{EE}$ & 0,059 & 0,021 & 0,040 & 0,020 \\
$\mathrm{p}$ & 0,0001 & 0,0001 & 0,0001 & 0,0001 \\
\hline
\end{tabular}

SA: semilla de algodón, SS: semilla de soja, $\mathrm{CN}$ : control sin suplemento, EE: error estándar, p: significancia, CT: colesterol total, TG: triglicéridos, C-HDL y C-LDL: colesterol de lipoproteínas de alta y baja densidad respectivamente. En cada columna letras distintas señalan diferencias significativas.

Tabla 4. Efectos del tratamiento y del tiempo sobre variables relacionadas al perfil lipídico de las vaquillas.

\begin{tabular}{cccccc}
\hline tratamiento & tiempo & $\mathrm{CT}(\mathrm{g} / \mathrm{l})$ & $\mathrm{TG}(\mathrm{g} / \mathrm{l})$ & $\begin{array}{c}\mathrm{C}-\mathrm{HDL} \\
(\mathrm{g} / \mathrm{l})\end{array}$ & $\begin{array}{c}\mathrm{C}-\mathrm{LDL} \\
(\mathrm{g} / \mathrm{l})\end{array}$ \\
\hline \multirow{2}{*}{$\mathrm{SA}$} & 1 & 2,09 & 0,40 & 1,45 & 0,43 \\
& 2 & 2,65 & 0,36 & 1,51 & 0,50 \\
$\mathrm{SS}$ & 1 & 1,87 & 0,39 & 1,30 & 0,38 \\
& 2 & 2,61 & 0,37 & 1,50 & 0,52 \\
$\mathrm{CN}$ & 1 & 1,45 & 0,22 & 0,95 & 0,22 \\
& 2 & 1,23 & 0,21 & 0,88 & 0,26 \\
$\mathrm{EE}$ & & 0,11 & 0,034 & 0,074 & 0,045 \\
$\mathrm{p}$ & & 0,0001 & 0,0001 & 0,0001 & 0,0001 \\
\hline
\end{tabular}

SA: semilla de algodón, SS: semilla de soja, CN: control sin suplemento, EE: error estándar, p: significancia, 1 y 2: primer y segundo inviernos, CT: colesterol total, TG: triglicéridos, C-HDL y C-LDL: colesterol de lipoproteínas de alta y baja densidad respectivamente.

Tabla 5. Efectos del tratamiento y del tiempo sobre las lipoproteínas de las vaquillas.

\begin{tabular}{|c|c|c|c|c|}
\hline tratamiento & tiempo & LA ( $\%)$ & LB (\%) & $\mathrm{BA}$ \\
\hline \multirow{4}{*}{ SA } & \multirow{2}{*}{ 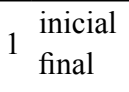 } & 87,42 & 12,58 & 0,15 \\
\hline & & 84,87 & 15,13 & 0,18 \\
\hline & \multirow{2}{*}{$2 \begin{array}{l}\text { inicial } \\
\text { final }\end{array}$} & 83,00 & 16,73 & 0,20 \\
\hline & & 88,60 & 11,13 & 0,13 \\
\hline \multirow{4}{*}{ SS } & \multirow{2}{*}{$\begin{array}{l}\text { inicial } \\
\text { final }\end{array}$} & 88,13 & 11,87 & 0,14 \\
\hline & & 85,21 & 14,79 & 0,18 \\
\hline & \multirow{2}{*}{$2 \begin{array}{l}\text { inicial } \\
\text { final }\end{array}$} & 81,17 & 18,83 & 0,24 \\
\hline & & 88,43 & 11,58 & 0,13 \\
\hline \multirow{4}{*}{$\mathrm{CN}$} & \multirow{4}{*}{$\begin{array}{ll}1 & \begin{array}{l}\text { inicial } \\
\text { final }\end{array} \\
2 & \begin{array}{l}\text { inicial } \\
\text { final }\end{array}\end{array}$} & 86,53 & 13,47 & 0,16 \\
\hline & & 81,03 & 18,98 & 0,24 \\
\hline & & 80,97 & 18,77 & 0,23 \\
\hline & & 88,00 & 12,00 & 0,14 \\
\hline \multicolumn{2}{|l|}{$\mathrm{EE}$} & 0,821 & 0,814 & 0,010 \\
\hline \multicolumn{2}{|l|}{$\mathrm{p}$} & 0,10 & 0,08 & 0,05 \\
\hline
\end{tabular}

SA: semilla de algodón, SS: semilla de soja, $\mathrm{CN}$ : control sin suplemento, EE: error estándar, p: significancia, 1 y 2 : primer y segundo inviernos, LA: lipoproteína alfa, LB: lipoproteína beta, BA: relación beta/alfa. tre tratamientos con altos y moderados tenores de grasa versus un control sin suplemento ${ }^{13}$.

En cuanto al nitrógeno no proteico, cabe destacar que el lote SS reveló $(\mathrm{p}=0,0002)$ mayores niveles séricos de urea $(0,45 \mathrm{~g} / 1)$ que los lotes $\mathrm{SA}(0,42 \mathrm{~g} / 1)$ y $\mathrm{CN}$ $(0,41 \mathrm{~g} / \mathrm{dl})$. En este caso parece existir una influencia de la grasa dietaria, tal como lo preconizan otros investigadores. Así, las concentraciones de urea sérica de vacas que recibieron dietas con moderada inclusión de lípidos $(0,11 \mathrm{~g} / \mathrm{l})$ fueron significativamente mayores que las sometidas a la dieta control $(0,09 \mathrm{~g} / \mathrm{l})$, pero no difirieron con las de animales que recibieron altos niveles de grasa $(0,11 \mathrm{~g} / \mathrm{l})$ durante el período de preparto. En cambio, en la fase postparto se registraron valores de urea de 0,14 $\mathrm{g} / \mathrm{l}$ en la dieta control versus $0,15 \mathrm{~g} / \mathrm{l}$ en las dietas con moderada y alta incorporación de grasa ${ }^{13}$.

En coincidencia con los resultados aquí obtenidos, la suplementación de vaquillas con semilla de algodón produjo elevación del nitrógeno ureico en los dos años en que se practicó (primer año: 0,11 vs $0,09 \mathrm{~g} / 1$ en testigos, $\mathrm{p}=0,05$ y segundo año: 0,086 vs $0,072 \mathrm{~g} / \mathrm{l}, \mathrm{p}=0,01$ ) ${ }^{29}$. Cabe recordar que el nitrógeno ureico en sangre es el parámetro habitualmente utilizado al momento de realizar ajustes en la administración de suplementos proteicos para ganado de carne en pastoreo ${ }^{19}$.

En la Tabla 3 se señalan las variaciones séricas de CT, TG, C-HDL y C-LDL para cada tratamiento. En la Tabla 4 se indica la influencia del efecto tiempo, que incidió sobre todas las variables lipídicas consideradas en la experiencia. Con relación al grupo control, CT aumentó significativamente $(p=0,0001)$ en ambos lotes suplementados. Ello era previsible desde el momento que el nivel sérico de $\mathrm{CT}$ guarda estrecha relación con el contenido de grasa ingerida por los animales ${ }^{12}$.

Elevaciones lineales de CT fueron verificadas en novillos que recibieron niveles crecientes de semilla de algodón (de 1,50 a 2,25 kg), implicando un aumento de 5,2 a $7,8 \%$ de grasas ingresadas al rumen ${ }^{22}$. Además de variar según las densidades calóricas (niveles de grasa) de las dietas suministradas a vacas lecheras, el CT aumenta al incrementarse la actividad ovárica, dado que es el precursor de las hormonas esteroides gonadales ${ }^{24}$. Así, elevaciones significativas del CT plasmático fueron registradas durante los períodos pre-parto y transicional, pero no en el post-parto de vacas lecheras ${ }^{13}$. Mayores concentraciones de TG y CT se registraron en vacas lecheras que recibieron grasas insaturadas en el pre-parto y saturadas en el post parto ${ }^{27}$.

Los niveles plasmáticos de TG aumentaron en SA y SS $(p=0,0001)$ tanto en el primero como segundo inviernos, aunque con cifras más bajas que las registradas en ensayos de suplementación energético-proteica anteriormente efectuados ${ }^{10}$. Cantidades crecientes de semilla de algodón en la dieta de novillos, produjeron elevaciones progresivas de TG plasmáticos $(0,34 ; 0,36$; $0,42$ y $0,69 \mathrm{~g} / 1)^{22}$. A la inversa, los niveles plasmáticos de TG disminuyeron al reducirse el aporte lipídico de la dieta en becerras ${ }^{24}$. 
Con relación al lote $\mathrm{CN}$, las tasas séricas de CHDL y C-LDL se incrementaron en SA y SS, siempre predominando C-HDL sobre C-LDL acorde al patrón lipoproteico de los rumiantes ${ }^{12}$. Similares aumentos fueron registrados en novillos suplementados con semilla de algodón y sorgo molido ${ }^{21,22}$. En concordancia, la administración de ácidos grasos en forma de jabones cálcicos redundó en incrementos séricos de HDL, VLDL, CT y TG ${ }^{14}$.

En la Tabla 5 se exponen los efectos del tratamiento y del tiempo sobre los valores de LA, LB y BA registrados a lo largo del ensayo, resaltando que el tiempo influyó significativamente sobre la concentración de las lipoproteínas séricas. En cuanto al tratamiento, surge que la tasa plasmática promedio de LA fue significativamente influenciada $(p=0,028)$ tanto por la suplementación con SA $(85,97 \%)$ como SS $(85,73 \%)$ en comparación al lote $\mathrm{CN}(84,13 \%)$. Inversamente, LB y BA fueron mayores en $\mathrm{CN}(15,81$ y $0,19 \%$ respectivamente) que en SA $(13,89$ y $0,16 \%$ respectivamente) y SS (14,27 y $0,17 \%$ respectivamente). Es generalizadamente admitido que en bovinos los niveles séricos de LA son fisiológicamente mayores que los de LB ${ }^{12,24}$. En otros ensayos de suplementación de distintas categorías bovinas con semilla de algodón, se hallaron descensos de los niveles de LB y BA, así como aumentos de LA, TG y CT 9,21,22.

La Tabla 6 muestra las modificaciones de algunas enzimas séricas indicadoras de disfunción hepática. Todas ellas fueron influenciadas por los efectos tratamiento y tiempo. AST reveló un significativo aumento $(p=0,0002)$ en los lotes SA y SS versus $C N$, de manera más manifiesta en el primer invierno (50, 45 y $42 \mathrm{UI} / 1)$ que en el segundo (32, 31 y $23 \mathrm{UI} / 1)$. Los valores aquí obtenidos se encontrarían dentro de los rangos de referencia admitidos (39 a $57 \mathrm{UI} / 1)^{12}$, ${ }^{16}$. En trabajos anteriores la actividad sérica de AST reveló progresivos incrementos séricos ante la incorporación de altos niveles de semilla de algodón en la dieta ${ }^{21,23}$.

La actividad media de GGT varió significativamente según el tratamiento $(\mathrm{p}=0,042)$, siendo de $23,22 \mathrm{y}$ $21 \mathrm{UI} / 1$ en los lotes SA, SS y CN respectivamente. LDH reveló marcado incremento en los lotes suplementados (SA: 1274 UI/1, SS: 1307 UI/l) con relación al lote control (1026 UI/l). En novillos suplementados con SA se observaron elevaciones significativas de AST, GGT y LDH, atribuibles a una leve sobrecarga hepática, con componente colestático y escasa inflamación ${ }^{30}$. Terneros que recibieron altos niveles de SA como suplemento, también registraron significativos aumentos de AST, GGT y LDH ${ }^{11}$.

En la Tabla 7 se registran las variaciones de los macro y micro elementos séricos investigados en la experiencia. Todos ellos fueron influenciados por el efecto tiempo. En cuanto al efecto tratamiento, la fosfatemia se manifestó significativamente elevada $(p=0,012)$ en
Tabla 6. Efectos del tratamiento sobre la actividad sérica de algunas enzimas de las vaquillas.

\begin{tabular}{cccc}
\hline tratamiento & AST $(\mathrm{UI} / 1)$ & GGT $(\mathrm{UI} / \mathrm{l})$ & $\mathrm{LDH}(\mathrm{UI} / \mathrm{l})$ \\
\hline $\mathrm{SA}$ & $41 \mathrm{a}$ & $23 \mathrm{a}$ & $1274 \mathrm{a}$ \\
$\mathrm{SS}$ & $38 \mathrm{a}$ & $22 \mathrm{ab}$ & $1307 \mathrm{a}$ \\
$\mathrm{CN}$ & $33 \mathrm{~b}$ & $21 \mathrm{~b}$ & $1026 \mathrm{~b}$ \\
$\mathrm{EE}$ & 1,654 & 0,823 & 18,748 \\
$\mathrm{p}$ & 0,002 & 0,042 & 0,0001 \\
\hline
\end{tabular}

SA: semilla de algodón, SS: semilla de soja, CN: control sin suplemento, EE: error estándar, p: significancia, AST: aspartato aminotransferasa, GGT: gamma glutamiltransferasa, LDH: lactato dehidrogenasa. En cada columna letras distintas señalan diferencias significativas.

Tabla 7. Efectos del tratamiento sobre algunos electrolitos séricos de las vaquillas.

\begin{tabular}{cccccccc}
\hline tratamiento & $\begin{array}{c}\text { calcio } \\
(\mathrm{mg} / \mathrm{dl})\end{array}$ & $\begin{array}{c}\text { fósforo } \\
(\mathrm{mg} / \mathrm{dl})\end{array}$ & $\begin{array}{c}\text { magnesio } \\
(\mathrm{mg} / \mathrm{dl})\end{array}$ & $\begin{array}{c}\text { sodio } \\
(\mathrm{meq} / \mathrm{l})\end{array}$ & $\begin{array}{c}\text { potasio } \\
(\mathrm{meq} / \mathrm{l})\end{array}$ & $\begin{array}{c}\text { hierro } \\
(\mathrm{ug} / \mathrm{dl})\end{array}$ & $\begin{array}{c}\text { cobre } \\
(\mathrm{ug} / \mathrm{dl})\end{array}$ \\
\hline $\mathrm{SA}$ & $9,63 \mathrm{a}$ & $6,76 \mathrm{a}$ & $2,59 \mathrm{a}$ & $142 \mathrm{a}$ & $5,24 \mathrm{a}$ & $145 \mathrm{a}$ & $112 \mathrm{a}$ \\
$\mathrm{SS}$ & $9,56 \mathrm{a}$ & $6,90 \mathrm{a}$ & $2,51 \mathrm{ab}$ & $142 \mathrm{a}$ & $5,11 \mathrm{ab}$ & $150 \mathrm{a}$ & $109 \mathrm{a}$ \\
$\mathrm{CN}$ & $9,35 \mathrm{a}$ & $6,34 \mathrm{~b}$ & $2,42 \mathrm{~b}$ & $140 \mathrm{~b}$ & $4,94 \mathrm{~b}$ & $135 \mathrm{~b}$ & $88 \mathrm{~b}$ \\
$\mathrm{EE}$ & 0,093 & 0,128 & 0,044 & 0,541 & 0,076 & 3,330 & 6,348 \\
$\mathrm{p}$ & 0,097 & 0,012 & 0,037 & 0,002 & 0,027 & 0,039 & 0,076 \\
\hline
\end{tabular}

SA: semilla de algodón, SS: semilla de soja, CN: control sin suplemen, EE: error estándar, p: significancia. En cada columna letras distintas erencias significativas

ambos períodos invernales, en los lotes SA y SS (6,76 y $6,90 \mathrm{mg} / \mathrm{dl}$ respectivamente) con relación al lote control $(6,34 \mathrm{mg} / \mathrm{dl})$. En el nordeste argentino los valores plasmáticos de fósforo inorgánico en bovinos sobre pasturas naturales suelen oscilar entre 4 y $5 \mathrm{mg} / \mathrm{dl}$, siendo más elevados en animales jóvenes ${ }^{12}$. La suplementación con SA generaría incrementos de la fosfatemia debido al elevado tenor fosfórico (1\%) contenido en tales semillas ${ }^{17}$. En ensayos anteriores se lograron significativos aumentos del fósforo inorgánico en sangre al emplear elevadas cantidades de SA en la ración: partiendo de valores iniciales de 4,5 mg/dl se llegó a niveles de 9,28 $\mathrm{mg} / \mathrm{dl}$ al cabo de 7 meses ${ }^{9}$.

Los valores de magnesio sérico hallados en esta experiencia concuerdan con los citados para el ganado de la zona $(2,4 \text { a } 3,3 \mathrm{mg} / \mathrm{dl})^{12}$. La magnesemia se mostró incrementada en los animales suplementados con SA $(\mathrm{p}=0,037)$. Este electrolito ostentó un mayor aumento en el segundo invierno $(2,62 ; 2,66$ y $2,44 \mathrm{mg} / \mathrm{dl}$ en $\mathrm{SA}$, SS y CN respectivamente), con relación al primero $(2,55 ; 2,36$ y $2,41 \mathrm{mg} / \mathrm{dl}$ respectivamente). Pese a que la absorción intestinal de magnesio sería afectada por la incorporación de elevados tenores de grasa a la ración ${ }^{25}$, en otros ensayos donde se empleó SA como suplemento, tampoco se registraron disminuciones de la magnesemia atribuibles al tratamiento ${ }^{9,21}$.

Sodio y potasio revelaron significativos aumentos en los animales que recibieron SA y SS ( $p=0,002$ y $0,027)$ versus los no suplementados. Igual comportamiento registró la ferremia (lote SS $150 \mathrm{ug} / \mathrm{dl}$, lote SA $145 \mathrm{ug} / \mathrm{dl}$ y lote CN $135 \mathrm{ug} / \mathrm{dl}$ ). Natremia y kalemia re- 
gistradas en esta experiencia permanecieron dentro del intervalo de referencia zonal (136-142 y 4,4-5,1 meq/1 respectivamente) ${ }^{12}$. No existiría deficiencia de $\mathrm{Na} \mathrm{ni}$ $\mathrm{K}$ en el suelo ni en las pasturas de la región donde se llevó a cabo el presente ensayo ${ }^{3}$. En otros trabajos, el estrés térmico incrementó significativamente $(\mathrm{p}<0,01)$ las concentraciones de potasio y calcio sérico en vacas Holstein y Jersey, aunque tales electrolitos disminuyeron $(p<0,01)$ en vacas cruza cebú afectadas por la misma noxa ${ }^{31}$.

El hierro tampoco sería deficiente en la región chaqueña donde se llevó a cabo la experiencia ${ }^{5}$. Consecuentemente, las ferremias aquí registradas fueron más elevadas que las consignadas en la bibliografía consultada $^{12}$. El nivel de hierro sérico es representativo del estatus férrico del organismo, como lo demuestran investigaciones efectuadas en terneros ${ }^{20}$.

Calcemias y cupremias no fueron influidas significativamente por los tratamientos. Los valores de calcio sérico se encontraron dentro de los rangos normales establecidos ${ }^{12}$. La calcemia no representaría las deficiencias dietarias (suelo, pastura, ración) porque los niveles sanguinos de este mineral son regulados hormonalmente ${ }^{2}$. Así, calcemia y fosfatemia no se vieron modificadas por la inclusión de diferentes proporciones de afrecho de trigo a la ración de novillos en engorde ${ }^{18}$. Los valores reportados en dicho trabajo (Ca: $13,63 \mathrm{mg}$ / $\mathrm{dl}, \mathrm{P}: 11,69 \mathrm{mg} / \mathrm{dl}$ ) son considerablemente más elevados que los hallados en el presente ensayo.

La cupremia registrada en esta experiencia se encontraría por encima de los valores citados en anteriores trabajos realizados en el nordeste argentino (86-97 $\mathrm{ug} / \mathrm{dl})^{12}$. Cabe destacar que el ganado del Chaco padece una grave deficiencia de este microelemento, debida al exceso de molibdeno en el suelo ${ }^{4}$.

En conclusión, surge que ambas semillas se comportaron como buenos suplementos nutricios invernales en vaquillas de recría cruza cebú (efecto tratamiento). La diferencia final de peso vivo entre ambos lotes tratados solamente fue de alrededor de $2 \mathrm{~kg}$ (no significativa), pero los controles sin suplementar permanecieron unos $20 \mathrm{~kg}$ por debajo (diferencia altamente significativa). El desarrollo de las vaquillas (ontogenia) y las variaciones climáticas y pastoriles dieron lugar a algunas diferencias entre los años 1 y 2 de ensayos (efecto tiempo). Entre las razas estudiadas no se registraron grandes diferencias de peso ni parámetros de laboratorio. Las vaquillas suplementadas revelaron favorables aumentos de variables de los perfiles eritrocitario, proteico, lipídico y mineral. Particularizando, la semilla de algodón indujo los más altos valores de hematocrito y magnesio (deseables), así como de colesterol total, AST y GGT (indeseables), en tanto que la semilla de soja generó los niveles más elevados de urea (deseable) y LDH (indeseable). Las elevaciones de lípidos y enzimas son relacionadas a una ligera sobrecarga hepática, la cual no repercutió en el estado general de salud de ningún animal. Tal ausencia de efectos secundarios de nivel clínico, aunada al aumento de peso y la mejoría de varios componentes del medio interno, autorizan la utilización de las semillas oleaginosas ensayadas como suplemento energético-proteico invernal de ganado en crecimiento en la zona chaqueña de Argentina.

\section{REFERENCIAS}

1. Baker RD. 1981. The herbage intake and performance of set-stocked suckler cows and calves. Grass \& Forage Sci 36: 210-214.

2. Balbuena O, Luciani CA, McDowell LR, Conrad JH, Martin FG. 1989. Estudios de la nutrición mineral de los bovinos para carne del este de las provincias de Chaco y Formosa (Argentina). 1. Fósforo y calcio. Vet Arg 6: 241253.

3. Balbuena O, McDowell LR, Toledo HO, Conrad JH, WiIkinson N, Mufarrege D. 1989. Estudios de la nutrición mineral de los bovinos para carne del este de las provincias de Chaco y Formosa (Argentina). 2. Magnesio, potasio y sodio. Vet Arg 6: 296-308.

4. Balbuena O, McDowell LR, Luciani CA, Conrad JH, Wilkinson N, Martin FG. 1989. Estudios de la nutrición mineral de los bovinos para carne del este de las provincias de Chaco y Formosa (Argentina). 3. Cobre, molibdeno y azufre. Vet Arg 6: 364-374.

5. Balbuena O, McDowell LR, Toledo HO, Conrad JH, WiIkinson N, Martin FG. 1989. Estudios de la nutrición mineral de los bovinos para carne del este de las provincias de Chaco y Formosa (Argentina). 4. Cinc, hierro y manganeso. Vet Arg 6: 584-594.

6. Balbuena O, Kudo H, Arakaki CL, Rochinotti D, Kucseva CD, Slanac AL, Koza GA, Schreiner JJ, Navamuel JM. 2004. Ruminal degradation of soybean with different processing methods. Anais III Congresso Mundial de Soja, Foz do Iguazú (Brasil), p. 289.

7. Banta JP, Lalman DL, Krehbiel CR, Wettemann RP. 2008. Whole soybean supplementation and cow age class: effects on intake, digestion, performance, and reproduction of beef cows. J Anim Sci 86: 1868-1878.

8. Cachapuz, JM. 1995. O panorama setorial da bovinocultura de corte gaúcha no processo de integração de Mercosul, $2^{\circ}$ ed., Ed. Emater, Porto Alegre, p. 68.

9. Coppo JA, Maccio OA, Scorza SH, Mussart NB. 1993. Desórdenes metabólicos en bovinos suplementados con semilla entera de algodón. Arch Zoot 42: 301-311.

10. Coppo JA, Scorza SH, Mussart NB. 1995. Biochemical profiles of argentine cattle supplemented with cottonseed. RIA 25: 91-102.

11. Coppo JA, Mussart NB. 2001. Efectos de la suplementación con semilla de algodón sobre el enzimograma plasmático de terneros destete. Actas de Ciencia y Técnica UNNE 4: 14-17.

12. Coppo JA. 2008. Fisiología comparada del medio interno, $2^{\circ}$ ed., EUCASA, Salta (Argentina), $310 \mathrm{p}$.

13. Douglas GN, Overton TR, Bateman HG, Drackley JK. 2004. Peripartal metabolism and production of Holstein cows fed diets supplemented with fat during the dry period. J Dairy Sci 87: 4210-4220. 
14. Espinoza JL, Ramirez-Godinez JA, Simental SS, Jiménez J, Ramirez R, Palacios A, Delun R. 1997. Effects of calcium soaps of fatty acids on serum hormones and lipid metabolites in Pelibuey ewes. Small Ruminant Res 26: 61-68.

15. Euclides VP. 2000. Alternativas para intensifição da produção de carne bovina em pastagem, Ed. Embrapa, Campo Grande (Brasil), p. 65.

16. Forchetti O. 2005. Valores de referencia de constituyentes bioquímicos sanguíneos en animales domésticos. Curso "Profundización y prácticas en métodos de laboratorio y diagnóstico veterinario", Ed. Fac. Agr. Vet. UNRC, Río Cuarto (Argentina).

17. Fumagalli AE. 2006. La semilla de algodón como suplementación en vacunos. On line: www.cuencarural.com/ ganaderia/bovinos/la_semilla_de_algodon.

18. Gendley MK, Singh P, Garg AK, Tiwari SP, Kumari K, Dutta GK. 2009. The studies on nutrient balances in crossbred cattle bulls fed chopped green sugarcane tops supplemented with some agro industrial by- products. Trop Anim Health Prod 41: 943-949

19. Hammond AC, Bowers EJ, Kunkle WE, Genho PC, Moore SA, Crosby CE, Ramsay KH. 1994. Use of blood urea nitrogen concentration to determine time and level of protein supplementation in wintering cows. Prof Anim Sci 10: 24-31.

20. Heidarpour Bami M, Mohri M, Seifi HA, Alavi Tabatabaee AA. 2008. Effects of parenteral supply of iron and copper on hematology, weight gain, and health in neonatal dairy calves. Vet Res Comm 32: 553-561.

21. Koza GA, Balbuena O, Mussart NB, Kusceva CD, Coppo JA. 2000. Efecto de diferentes niveles de semilla de algodón en la dieta sobre algunos metabolitos sanguíneos de novillos. Rev Arg Prod Anim 20: 66-67.

22. Koza GA, Balbuena O, Mussart NB, Kusceva CD, Coppo JA. 2001. Cambios del lipidograma en novillos suplementados con semilla de algodón. On line: http://www. unne.edu.ar/cyt/2001/cyt.htm.
23. Koza GA, Mussart NB, Coppo JA. 2005. Evolución de algunos indicadores del metabolismo lipídico en novillos suplementados con distintos niveles de semilla de algodón. Anales de la XXVI Sesión de Comunicaciones Cientificas, Facultad de Ciencias Veterinarias, UNNE (Corrientes, Argentina), p. 66.

24. López Ortega AA, Márquez YC, Mendoza CA, Ferraro SM, Márquez AA. 2008. Perfil lipídico en becerras mestizas Carora durante el primer año de vida, en época de lluvias y de sequía, en Venezuela. Rev vet 19: 2-7.

25. Palmquist DL. 1996. Utilización de lípidos en dietas de rumiantes. Anales XII Curso de especialización FEDNA (Fund Españ Desarr Nutr Anim), Madrid, 15 p. On line: http://www.etsia.upm.es/fedna/capitulos/96capituloIII. pdf.

26. Peruchena CO. 1997. Dietas para la nutrición de bovinos en crecimiento y engorde en la región subtropical, Boletín Prod Anim INTA (Corrientes, Argentina), p.13.

27. Petit HV, Palin MF, Doepel L. 2007. Hepatic lipid metabolism in transition dairy cows fed flaxseed. J Dairy Sci 90: 4780-4792

28. Poore MH, Scott ME, Green JT. 2006. Performance of beef heifers grazing stockpiled fescue as influenced by whole cottonseed supplemental. J Anim Sci 84: 16131625.

29. Rebollar PG, De Blas C. 2004. Digestión de la soja integral en rumiantes. On line: http://www.asa- europe.org/ library_e.shtml/animalnutrition.

30. Slanac AL, Mussart NB, Coppo JA. 1997. Efectos de la suplementación con semilla de algodón sobre el enzimograma plasmático de novillos cruza cebú. Anales III Reunión Latinoam. Fisiol. Vet. (Piriápolis, Uruguay), p. 6.

31. Srikandakumar A, Johnson EH. 2004. Effect of heat stress on milk production, rectal temperature, respiratory rate and blood chemistry in Holstein, Jersey and Australian Milking Zebu cows. Trop Anim Health Prod 36: 685692. 